\title{
Identification of latent tuberculosis infection in rheumatic patients under consideration for treatment with anti-TNF- $\alpha$ agents
}

Jolanta Paluch-Oleś1, Agnieszka Magryś, Maria Kozioł-Montewka1, Arkadiusz Koszarny², Maria Majdan²

1Department of Medical Microbiology, Medical University of Lublin, Poland 2Department of Rheumatology and Connective Tissue Diseases, Medical University of Lublin, Poland

Submitted: 10 January 2011

Accepted: 9 June 2011

Arch Med Sci 2013; 9, 1: 112-117

DOI: 10.5114 /aoms.2013.33352

Copyright (c) 2013 Termedia \& Banach

\begin{abstract}
Introduction: Immunosuppressive therapy with anti-tumour necrosis factor- $\alpha$ (TNF- $\alpha$ ) agents in rheumatic patients modulates the immune system and may increase the risk of reactivating infections that are normally maintained in a latent state, such as tuberculosis. The purpose of this study was to analyse the value of QuantiFERON TB Gold In-Tube (QFT IT) and tuberculin skin test (TST) in BCG vaccinated patients with rheumatoid arthritis and ankylosing spondylitis who were qualified to receive TNF- $\alpha$ blockers.

Material and methods: Ninety patients with rheumatoid arthritis and ankylosing spondylitis were included in the study. The control group consisted of 20 healthy participants. Chest X-ray, TST and QFT IT were carried out in all persons.

Results: In rheumatic patients positive results of QFT IT and TST tests were identified in 15 cases $(16.7 \%)$ whereas negative results of both tests were detected in 56 cases (62.2\%). In the group of examined patients, 11 (12.2\%) had QFT IT-/TST+ test results. In patients with QFT IT+/TST- status one active tuberculosis case was detected. In the control group QFT IT positive results were found in 4 cases (20\%) and TST positive in 11 cases (55\%). Treatment with TNF- $\alpha$ blockers was introduced in 26 rheumatology patients with the following test status: 3 with QFT IT+/TST+; 20 with QFT IT-/TST-; 3 with QFT IT-/TST+.

Conclusions: In the BCG vaccinated population the QFT IT assay may potentially improve the identification and selection for therapy for latent TB infection before treatment with anti-TNF agents.
\end{abstract}

Key words: QuantiFERON-TB Gold In-Tube, tuberculin skin test, rheumatoid arthritis, latent tuberculosis, anti-TNF- $\alpha$ therapy.

\section{Introduction}

Rheumatoid arthritis (RA) and ankylosing spondylitis (AS) are chronic, progressive, and painful inflammatory rheumatic diseases that affect approximately $1 \%$ of the world population. The joint damage in these diseases is mediated through an immunological mechanism with tumour necrosis factor- $\alpha$ (TNF- $\alpha$ ) as a particularly important cytokine [1, 2].

The introduction of TNF- $\alpha$ blocking agents has been a major breakthrough in the therapy of these chronic inflammatory diseases [3].

The immunosuppressive therapy with anti-TNF- $\alpha$ agents modulates the immune system, so it may increase the risk of reactivating infections that are normally maintained in a latent state such as tuberculosis [4, 5]. It is

\author{
Corresponding author: \\ Agnieszka Magryś PhD \\ Department of Medical \\ Microbiology \\ Medical University of Lublin \\ 1 Chodzki Str. \\ 20-093 Lublin, Poland \\ Phone/fax: +48 817423781 \\ E-mail: magrysa@gmail.com
}


universally accepted that patients who are being considered for treatment with anti-TNF- $\alpha$ agents should be evaluated for the presence of active tuberculosis and latent tuberculosis infection (LTBI) before the introduction of biological therapy $[3,4]$. Until recently, screening of these patients included their history of exposure to Mycobacterium tuberculosis (MTB), a tuberculin skin test (TST) and a chest X-ray [3].

Although diagnosis of LTBI still widely relies on the TST, the accuracy of the test in rheumatic patients is limited because of the presence of an underlying disease and immunosuppressive therapy [6-8]. Moreover, in countries like Poland whose population is universally immunized with the BCG vaccine, the TST may give false-positive results.

More recently, a new diagnostic method that can overcome the limitations of TST has been developed: QuantiFERON-TB Gold In-Tube (QFT IT). The assay quantifies interferon $-\gamma($ IFN $-\gamma)$ released by $T$ cells after the stimulation by MTB antigens. The IFN- $\gamma$ test is highly specific and unaffected by prior $B C G$ vaccination or immune reactivity to most atypical mycobacteria. It is also more sensitive than the TST in detecting people with latent tuberculosis infection.

The aim of the present work was to analyse the utility of MTB-specific QuantiFERON-TB Gold InTube and TST in patients with rheumatoid arthritis and ankylosing spondylitis who were qualified to receive TNF- $\alpha$ blocking agents.

\section{Material and methods}

\section{Patients}

Ninety patients from the Department of Rheumatology and Connective Tissue Diseases in Lublin, Poland, who suffered from rheumatoid arthritis (RA, $n=81$ ) and ankylosing spondylitis (AS, $n=9$ ) were included in the study. All patients enrolled in the study met the criteria of the American College of Rheumatology for classification of acute arthritis and ankylosing spondylitis $[9,10]$. The study was conducted between December 2007 and December 2008. For each patient we gathered demographic information together with clinical characteristics of RA or AS. The mean age of the patients was 53 years (ranging from 19 to 82 years). The randomly assigned control group consisted of 20 healthy participants (mean age: 38.9 years). Chest $X$-ray, TST and QFT IT were carried out in patient and control groups. The QFT IT was performed one week after TST results were obtained. Table I summarizes the main features of patient and control groups.

\section{Qualification for biological treatment with TNF- $\alpha$ blocking agents}

Patients with i) established severe active RA or $A S$, ii) persistent symptoms and signs of active dis- ease and those who iii) failed adequate therapy with conventional disease-modifying anti-rheumatic drugs (DMARD) were qualified to receive antiTNF- $\alpha$ therapy with either infliximab (INFL), etanercept (ET) or adalimumab (ADA) with standard doses.

\section{Diagnosis of latent tuberculosis infection}

The presence of LTBI was assessed by medical history and screening tests: chest radiography, TST and QFT IT. The evaluation of the medical history included: current symptoms, prior history of treatment for tuberculosis and close contact with active pulmonary tuberculosis within the last year.

Tuberculin skin test: The TST was performed on the volar surface of the forearm according to the Mantoux method. A 2 TU dose $(0.1 \mathrm{ml})$ of tuberculin purified derivative (PPD RT23; Statens Serum Institut, Copenhagen, Denmark) was injected intracutaneously and the induration was measured after

Table I. Patients' characteristics

\begin{tabular}{|c|c|c|}
\hline Features & $\begin{array}{l}\text { Study group } \\
\qquad(N=90)\end{array}$ & $\begin{array}{c}\text { Control group } \\
(N=20)\end{array}$ \\
\hline \multicolumn{3}{|l|}{ Gender, $n(\%):$} \\
\hline Male & $23(25.5)$ & $7(35.0)$ \\
\hline Female & $67(74.4)$ & $13(65.0)$ \\
\hline Age [years], mean (range) & $53.1(19-82)$ & $38.9(26-58)$ \\
\hline \multicolumn{3}{|l|}{ Primary disease, $n(\%)$ : } \\
\hline $\mathrm{RA}$ & $81(90)$ & - \\
\hline AS & $9(10)$ & - \\
\hline \multicolumn{3}{|l|}{ Previous TB treatment, $n$ (\%): } \\
\hline Yes & $2(2.2)$ & - \\
\hline No & $88(97.7)$ & - \\
\hline \multicolumn{3}{|l|}{ Close TB contact, $n(\%)$ : } \\
\hline Yes & $1(1.1)$ & $0(0.0)$ \\
\hline No & $89(98.8)$ & $20(100.0)$ \\
\hline \multicolumn{3}{|l|}{ Chest X-ray, $n(\%):$} \\
\hline Positive & $5(5.6)$ & $0(0.0)$ \\
\hline Negative & $85(94.4)$ & $20(100.0)$ \\
\hline \multicolumn{3}{|l|}{ TST results ${ }^{*}, * *, n(\%):$} \\
\hline Positive & $26(28.9)$ & $11(55.0)$ \\
\hline Negative & $64(71.1)$ & $9(45.0)$ \\
\hline \multicolumn{3}{|l|}{ QFT results ${ }^{*, * *}, n(\%):$} \\
\hline Positive & $20(22.2)$ & $4(20.0)$ \\
\hline Negative & $67(74.7)$ & $16(80.0)$ \\
\hline Indeterminate & $3(3.3)$ & $0(0.0)$ \\
\hline
\end{tabular}

*Total agreement between the QFT IT and TST in study group $=0.82$ (0.95 Cl: $0.7155-0.8881), \kappa=0.53$ (0.95 Cl: $0.3306-0.7294)$, * total agreement between the QFT IT and TST in control group $=0.35(0.95 \mathrm{Cl}$ : 0.1631-0.5905), $\mathrm{\kappa}$ is not calculated 
$72 \mathrm{~h}$, with $5 \mathrm{~mm}$ diameter used as the cut-off for a positive test result in the patient group and $10 \mathrm{~mm}$ in the control group [11].

QuantiFERON-TB Gold In-Tube test: The test was performed according to the manufacturer's recommendations. Briefly, the test was performed in two stages. In the first stage, whole heparinized blood was collected into tubes with ESAT-6, CFP-10 and TB7.7 antigens as well as T-cell mitogen phytohaemagglutinin and a negative control and incubated for $16 \mathrm{~h}$ to $24 \mathrm{~h}$. After centrifugation the plasma samples were harvested.

In the second stage, plasma samples and conjugate were loaded onto a QFT ELISA plate and the detection of IFN- $\gamma$ levels was performed simultaneously with duplicate kit standards. Substrate reagent was added after $2 \mathrm{~h}$ incubation, Stop Solution was added $0.5 \mathrm{~h}$ later, then the OD values of all samples were read on an ELISA Multiscan reader (Labsystem, Finland). The results were interpreted using QFT Analysis Software. The result was considered positive when the concentration of IFN- $\gamma$ was greater than or equal to $0.35 \mathrm{IU} / \mathrm{ml}$ and negative when it was less than $0.35 \mathrm{IU} / \mathrm{ml}$ after subtraction of the respective nil value. Apart from this, an indeterminate result was generated when the IFN- $\gamma$ concentration in the TB antigen was less than $0.35 \mathrm{IU} / \mathrm{ml}$ and less than $0.5 \mathrm{IU} / \mathrm{ml}$ in the mitogen tube after subtraction of the respective nil value.

\section{Statistical analysis}

For statistical analysis the weighted $\kappa$ coefficient was used where a $\kappa$ value $=0$ is classified as poor agreement; $\kappa$ values from 0.01 to 0.20 constitute slight agreement; from 0.21 to 0.40 constitute fair agreement; from 0.41 to 0.60 constitute moderate agreement; from 0.61 to 0.80 constitute substan- tial agreement; and $\kappa$ values $\geq 0.81$ constitute perfect agreement. For statistical analysis results were expressed as the mean $95 \%$ confidence intervals $(95 \% \mathrm{Cl})$.

\section{Results}

The study group characteristics are summarized in Table I. A total of 67 female and 23 male patients with a mean age of 53.1 years were enrolled in the patient group. Of 90 patients $81(90 \%)$ had a diagnosis of RA and the remaining $9(10 \%)$ had AS. Eighty-two patients were selected for treatment with TNF inhibitors, and 8 patients were undergoing treatment with anti-TNF- $\alpha$ blocking agents. Five patients had chest $X$-ray findings suggestive of pulmonary tuberculosis. One of them had recent close contact with a patient with active TB.

The TST and QFT IT tests were performed in all cases. Twenty (22.2\%) patients had positive QFT IT results. The TST was found positive for 26 (28.9\%) patients. The total agreement between the QFT IT and TST test results was 0.82 . Cohen's $\kappa$ coefficient was moderate, with a value of 0.53 . In the control group QFT IT positive results were found in 4 cases (20\%) and TST positive in 11 cases (55\%). Total agreement between the QFT IT and TST in the control group $=0.35 ; \kappa$ was not calculated. In rheumatic patients the positive results of both tests were concordant in 15 (16.7\%) cases (Table II). Among these 15 patients 3 started biological therapy with ET (Table II). Twelve patients did not qualify for biological treatment. Of these patients one person was diagnosed with active TB and the remaining 11 were directed for diagnostics of tuberculosis (Tables II, III).

The negative results of QFT IT and TST tests were concordant in 56 (62.2\%) cases (Table II). Twenty

Table II. Treatment of rheumatic diseases according to test results $(N=90)$

\begin{tabular}{|c|c|c|c|c|c|}
\hline & \multicolumn{5}{|c|}{ Biological treatment } \\
\hline & \multicolumn{4}{|c|}{ Yes } & \multirow[t]{2}{*}{ No } \\
\hline & INFL & ET & ADA & Total & \\
\hline QFT+/TST+, $n=15$ (16.7\%) & - & $3^{*}(3.3 \%)$ & - & $3(3.3 \%)$ & $12(13.3 \%)$ \\
\hline QFT-/TST-, $n=56(62.2 \%)$ & $7(7.8 \%)$ & $7(7.8 \%)$ & $6(6.7 \%)$ & $20(22.2 \%)$ & $36(40.0 \%)$ \\
\hline QFT-/TST+, $n=11$ (12.2\%) & $1(1.1 \%)$ & $2(2.2 \%)$ & - & $3(3.3 \%)$ & $8(8.8 \%)$ \\
\hline QFT+/TST-, $n=5(5.6 \%)$ & - & - & - & - & $5(5.6 \%)$ \\
\hline QFT inde/TST-, $n=3(3.3 \%)$ & - & - & - & - & $3(3.3 \%)$ \\
\hline Total & $8(8.9 \%)$ & $12(13.3 \%)$ & $6(6.7 \%)$ & $26(28.9 \%)$ & $53(58.8 \%)$ \\
\hline $\begin{array}{l}\text { Cohen's } \kappa \text { coefficient, } \\
\text { agreement }\end{array}$ & & & & $\begin{array}{c}\kappa=0.61 \\
(0.95 \mathrm{Cl}: \\
0.2245-0.9929) \\
\text { agreement }=0.88 \\
(0.95 \mathrm{Cl}: \\
0.7741-0.9626)\end{array}$ & $\begin{array}{c}\kappa=0.39 \\
(0.95 \mathrm{Cl}: \\
0.1242-0.6654) \\
\text { agreement }=0.74 \\
(0.95 \mathrm{Cl}: \\
0.5939-0.8492)\end{array}$ \\
\hline
\end{tabular}

*treatment of $L T B I$ concomitantly 
patients were qualified for biological treatment: 7 with INFL, 7 with ET, 6 with ADA. In 36 (40.0\%) cases biological treatment was not taken into consideration (Table II).

In the analysed group of patients with rheumatological diseases, 11 had QFT IT-/TST+ test results. Even though the TST results were positive in 3 patients they received biological treatment: 1 with INFL and 2 with ET. These patients did not have recent contact with active TB patients, they did not have abnormal chest X-rays suggesting LTBI, nor were they inefficiently treated for tuberculosis in the past.

In the group of patients with QFT IT+/TST- status ( $n=5 ; 5.6 \%)$ none started biological treatment as one active tuberculosis case was detected and the remaining 4 patients were directed for microbiological evaluation of TB (Tables II and III). For three patients with indeterminate QFT IT and negative TST results a treatment other than biological was introduced (Table II).

In the group of patients qualified for biological treatment the $\kappa$ coefficient was substantial, with a value of 0.61 , in comparison to the group of patients who were not qualified for biological treatment, with substantial agreement of 0.74 and $\kappa=0.39$ (Table II).

\section{Discussion}

The proinflammatory cytokine TNF- $\alpha$ plays a key role in the pathogenesis of some rheumatic diseases such as RA and AS but also has a critical role in the host's immune response against MTB. Since TNF- $\alpha$ has a major role in sequestering tuberculous bacilli in granuloma and preventing their dissemination, using TNF blocking agents may result in the reactivation of $L T B I$. It is confirmed that there is a 4-8-fold increased risk of TB reactivation in patients taking agents that antagonize the biological role of TNF- $\alpha$ and more than 5 -fold increased risk in patients on steroid therapy [4].

On the other hand, using anti-TNF- $\alpha$ drugs is the most effective method in treatment of RA or AS [6]. Active TB or reactivation of LTBI always presents a critical and serious complication in patients receiving TNF- $\alpha$ inhibitors. Therefore, it is a challenge in immunocompromised patients to rule out LTBI before anti-TNF- $\alpha$ therapy is started [4].

In Poland tuberculosis incidence rates are among the highest in the European Union but incidences are decreasing. In 2008 an incidence rate of 21.2 per 100000 population was reported. The prevalence of LTBI is unknown largely due to lower diagnostic value of the skin test in a population that is universally immunized for BCG and has had multiple TST examinations [12]. In our study we observed $55 \%$ of healthy individuals with TST positive results, whereas only $20 \%$ were positive by QFT IT. Such discordance could be due to previous BCG vaccination.
Table III. Identification and treatment of tuberculosis among patients not classified for biological treatment

\begin{tabular}{|lcc|}
\hline \multirow{2}{*}{$\begin{array}{l}\text { Not classified for biological } \\
\text { treatment, } N=53 \text { (58.8\%) }\end{array}$} & \multicolumn{2}{c|}{ Tuberculosis } \\
\cline { 2 - 3 } & TB diagnostics & Active TB \\
\hline $\begin{array}{l}\text { QFT+/TST+ } \\
n=12(13.3 \%)\end{array}$ & $11(12.2 \%)$ & $1(1.1 \%)$ \\
\hline $\begin{array}{l}\text { QFT-/TST- } \\
n=28(31.1 \%)\end{array}$ & - & - \\
\hline $\begin{array}{l}\text { QFT-/TST+ } \\
n=8(8.8 \%)\end{array}$ & $2(2.2 \%)$ & - \\
\hline $\begin{array}{l}\text { QFT+/TST- } \\
n=5(5.6 \%)\end{array}$ & $4(4.4 \%)$ & \\
\hline $\begin{array}{l}\text { QFT inde/TST- } \\
n=3(3.3 \%)\end{array}$ & - & - \\
\hline Total & $14(15.6 \%)$ & $2(2.2 \%)$ \\
\hline
\end{tabular}

Until recently, clinical rheumatology practice required negative TST before beginning biological therapy. However, the utility of this test for the diagnosis of LTBI has been questioned due to the high rate of false results. The TST interpretation is specifically difficult in immunosuppressed individuals, such as those with RA or AS, taking steroids and/or DMARD. For these reasons, the Polish Rheumatology Experts Group's proposal of recommendations for prophylaxis and management of tuberculosis in patients treated with TNF- $\alpha$ antagonists suggests IFN- $\gamma$-release assays, such as QFT IT instead of TST, together with chest radiography and medical history for the diagnosis of LTBI [13]. The suggestions have been obligatory in Poland since 2008.

In our study 20 (22.2\%) patients were considered to have LTBI as detected on the basis of positive QFT IT and 26 (28.9\%) were TST positive. In comparison, Cobanoglu et al. [14] from Turkey reported that $10.3 \%$ of rheumatic patients, all BCG vaccinated, had positive QFT results while $50.5 \%$ were TST positive. Similarly to Poland, Turkey is a country with a relatively high prevalence of tuberculosis with a total incidence rate of 25/100 000 in 2007. In contrast, in the study of Pratt et al. [15] from a low prevalence country like the UK (13 per 100000 in 2007) $6.9 \%$ of RA patients were QFT positive.

Among the 90 patients for whom QFT IT and TST were used for LTBI detection there was $82 \%$ total agreement between the two tests. Accordingly, the $\kappa$ statistic between QFT IT and TST was moderate, with a value of 0.53 . In the Turkish study of Inanc et al. performed in RA patients from a BCG vaccinated population the total agreement of $61 \%$ with kappa coefficient 0.29 between QFT IT and TST results was obtained [16].

QFT IT confirmed the positive TST results in 15 cases $(16.7 \%)$. In $16(n=11$ and $n=5)$ cases the re- 
sults of QFT IT and TST were discordant. Among them there could be false positive as well as false negative test results. In Polish patients, false positive TST results are most likely due to cross reactivity to BCG or boosting effects as everybody born after 1955 had to be vaccinated with BCG against TB [17]. Furthermore, false negative TST results are frequently associated with long-term immunosuppressive treatment with DMARD. False negative TST results are particularly dangerous for patients on biological treatment because of the progression of LTBI to TB in a short time. In our study it was shown as the difference in number of negative TST results between patient and control groups ( $71.1 \%$ vs. $45 \%$, respectively). All the facts support the usefulness of IFN- $\gamma$-releasing assays for screening purposes in rheumatological patients [18]. QFT IT may yield indeterminate results in immune suppressed patients taking steroids or DMARD but does not occur frequently (5-13\%) [19]. In our study 3 (3.3\%) patients had indeterminate QFT IT results. These patients were initially treated with gold salts, DMARD and steroids for an average of 10 years. At the time of the study the patients were treated with methotrexate, leflunomide and $5 \mathrm{mg}$ prednisone. Such treatment may cause immunodeficiency resulting in T-cell anergy. The indeterminate QFT IT results are important diagnostic indicators, unlike TST, which is either positive or negative without information about patients' immune status [20]. Moreover, the higher diagnostic accuracy of QFT IT, including the availability of internal positive and negative controls, is a relevant advantage of the test.

Rheumatoid arthritis and ankylosing spondylitis are chronic rheumatic diseases so not only is QFT IT useful at the initial decision to treat with biological treatment, but also the subsequent therapy is often dependent on the result of the test. In our setting QFT IT was performed for the first time in patients during biological treatment. It was undertaken to avoid the risk of reactivating a latent infection to become an active disease in cases of false negative TST results as well as the burden of unnecessary treatment of LTBI in cases of false positive TST results. In our study, in 2 patients with previous false negative TST results treatment was discontinued because of QFT IT positivity. QFT IT should be the test of choice to eliminate false positive TST results due to BCG vaccination and to reduce the number of false negative results due to immune suppression in this cohort of patients [4].

In conclusion, as there is a lack of gold standard tests for LTBI detection, it is difficult to ascertain the true sensitivity and specificity of QFT IT. Our findings suggest that QFT IT may be helpful for screening purposes in patients prior to anti-TNF- $\alpha$ therapy. In the Polish population the QFT IT assay seemed to be a more accurate test for the detection of LTBI in rheumatic patients compared with TST before the treatment with anti-TNF agents. Moreover, the introduction of QFT IT in populations with high incidence rates of tuberculosis increases the possibility of active TB identification among patients with LTBI. To sum up, the QFT IT test can be used for LTBI detection in BCG-vaccinated rheumatic patients and can successfully replace the TST.

\section{References}

1. Malaviya AM. Cytokine network and its manipulation in rheumatoid arthritis. J Assoc Physicians India 2006; 56: 15-8.

2. Caporali R, Pallavicini FB, Filippini M, et al. Treatment of rheumatoid arthritis with anti-TNF-alpha agents: a reappraisal. Autoimm Rev 2009; 8: 274-80.

3. Keane J. TNF-blocking agents and tuberculosis: new drugs illuminate an old topic. Rheumatology 2005; 44: 714-20.

4. Whintrop KL. Risk and prevention of tuberculosis and other serious opportunistic infections associated with the inhibition of tumor necrosis factor. Nat Clin Pract Rheumatol 2006; 2: 602-10.

5. Vassilopoulos D, Stamoulis N, Hadziyannis E, Archimandritis Al. Usefulness of enzyme-linked immunospot assay (Elispot) compared to tuberculin skin testing for latent tuberculosis screening in rheumatic patients scheduled for anti-tumor necrosis factor treatment. J Rheumatol 2008; 35: 1271-6.

6. Hanta I, Ozbek S, Kuleci S, Kocabas A. The evaluation of latent tuberculosis in rheumatologic diseases for anti-TNF therapy: experience with 192 patients. Clin Rheumatol 2008; 27: 1083-6.

7. Nizam S, Emery P. Attenuated response to purified protein derivative in patients with rheumatoid arthritis. Ann Rheum Dis 2006; 65: 980.

8. Emery P, Panayi G, Symmons D, Brown G. Mechanisms of depressed delayed-type hypersensitivity in rheumatoid arthritis: the role of protein energy malnutrition. Ann Rheum Dis 1984; 43: 430-4.

9. Arnett F, Edworthy S, Bloch D, et al. The American Rheumatism Association 1987 revised criteria for classification of rheumatoid arthritis. Arthritis Rheum 1988; 31: 315-24.

10. van der Linden S, Valkenburg HA, Cats A. Evaluation of diagnostic criteria for ankylosing spondylitis. A proposal for modifications of New York criteria. Arthritis Rheum 1984; 27: 361-8.

11. CDC. Diagnostic standards and classification of tuberculosis in adults and children. Am J Respir Crit Care Med 2000; 161: 1376-95.

12. Demkow U, Broniarek-Samson B, Filewska M, et al. Prevalence of latent tuberculosis infection in health care workers in Poland assessed by interferon-gamma whole blood andtuberculin skin tests. J Physiol Pharmacol 2008; 59 Suppl. 6: 209-17.

13. Kucharz E, Korzeniewska-Koseła M, Kotulska A. Recommendations for prophylaxis and management of tuberculosis in patients treated with TNF-alpha antagonists. Reumatologia 2008; 46: 51-4.

14. Cobanoglu N, Ozcelik U, Kalyoncu U, et al. Interferongamma assays for the diagnosis of tuberculosis infection before using tumour necrosis factor alpha blockers. Int J Tuberc Lung Dis 2007; 11: 1177-82. 
15. Pratt A, Nicholl K, Kay L. Use of QuantiFERON TB Gold test as part of screening programme in patients with RA under consideration for treatment with anti-TNF-alpha agents: the Newcastle (UK) experience. Rheumatology 2007; 46: 1035-6.

16. Inanc N, Aydin Sz, Karakurt S, Atagunduz P, Yavuz S, Direskeneli H. Agreement between Quantiferon-TB gold test and tuberculin skin test in the identification of latent tuberculosis infection in patients with rheumatoid arthritis and ankylosing spondylitis. J Rheumatol 2009; 36: 2675-81.

17. Szczuka I. Bezpieczeństwo szczepień BCG - niepożądane odczyny poszczepienne. Przegl Epidemiol 2002; 56: 1-13.

18. Bocchino M, Matarese A, Bellofiore B, et al. Performance of two commercial blood IFN-gamma release assays for the detection of Mycobacterium tuberculosis infection in patient candidates for anti-TNF-alpha treatment. Eur J Clin Microbiol Infect Dis 2008; 27: 907-13.

19. Kobashi Y, Mouri K, Obase Y, Fukuda M, Miyashita N, Oka M. Clinical evaluation of QuantiFERON TB-2G test for immunocompromised patients. Eur Respir J 2007; 30: 945-50.

20. Matulis G, Jüni P, Viliger PM, Gadola SD. Detection of latent tuberculosis in immunosuppressed patients with autoimmune diseases: performance of a Mycobacterium tuberculosis antigen-specific interferon gamma assay. Ann Rheum Dis 2008; 67: 84-90. 